\title{
Genetic associations with RA expanded and strengthened
}

By genotyping 186 loci, Eyre et al. have identified 14 new genetic associations with rheumatoid arthritis (RA), taking the total number of known susceptibility loci for this disease to 46. Furthermore, previously identified RA risk loci were confirmed, many were linked to a single gene, and a number of potential causal polymorphisms were identified.

The study was a large collaborative effort, involving many groups around the world. Together, they used a singlenucleotide polymorphism (SNP) array to investigate 129,464 markers in 11,475 individuals of European ancestry with RA and 15,870 healthy controls. The array was developed by the Immunochip consortium to contain all known SNPs for the 186 loci tested, which were chosen based on their association with 12 autoimmune diseases.

Of the patients with RA enrolled, 7,222 were confirmed as anti-citrullinated peptide antibody (ACPA) positive and 3,297 were ACPA negative. In addition, data for a further 2,363 $\mathrm{ACPA}^{+}$patients with RA and 17,872 controls from previous genome-wide association studies were combined with the Immunochip data in a meta-analysis. This approach enabled the identification of 5 new susceptibility loci specifically for $\mathrm{ACPA}^{+}$ disease. Such associations suggest that different SNPs might predispose an individual to $\mathrm{ACPA}^{+} \mathrm{RA}$ rather than $\mathrm{ACPA}^{-}$disease.

\section{4 ...most of the loci associated with RA contain genes with immunological roles... 77}

The newly discovered susceptibility loci only raise the estimated heritability of RA from $47 \%$ to $51 \%$, with HLA accounting for $36 \%$. Fine mapping of the $\mathrm{MHC}$ region using the Immunochip assay confirmed previous associations with $\mathrm{ANCA}^{+} \mathrm{RA}$, the strongest corresponding to SNPs affecting amino acids 11,71 , and 74 , which line the peptide binding groove. Thus, these data add weight to the shared epitope hypothesis of RA causality.
Dense SNP coverage of 39 non-HLA loci linked to RA localised the association to a specific gene for 19; seven possibly causal exonic SNPsincluding one in $I L 6 R$, which encodes the target of tocilizumab-and others with potential regulatory activity were identified. Importantly, most of the loci associated with RA contain genes with immunological roles. Indeed, the authors found that the risk loci contain genes expressed predominantly by $\mathrm{CD} 4^{+}$ effector memory $\mathrm{T}$ cells.

These findings advance our understanding of the etiology of RA and identify genetic variations underlying different disease subtypes. This

knowledge could enable the development of novel effective drugs and stratification of therapy.

David Killock

Original article Eyre, S. et al. High-density genetic mapping identifies new susceptibility loci for rheumatoid arthritis. Nat. Genet. doi:10.1038/ng.2462 